\title{
Timetable management and operational simulation: methodology and perspectives
}

\author{
A. Radtke \\ Institut für Verkehrswesen, Eisenbahnbau und-betrieb, \\ University of Hannover, Germany
}

\begin{abstract}
This article summarizes the fundamental basics for the timetable management of the future. The advantages of a microscopic database and some options for its smart utilisation are shown. After describing some important areas of application of the microscopic methodology, some practical experiences are discussed. The "Seven-Step-Model" is a first attempt to provide a methodology for using microscopic models. The description of the process highlights the iterative approach. The presented methodology can be used for the evaluation of the effort and time requirements for various planning tasks. The article closes with an outlook and a discussion about solving the problems of the planning task in the future.
\end{abstract}

Keywords: methodology of planning of railway operation, timetable construction, operational railway simulation.

\section{Introduction}

The timetable management is a key process for any railway company (RC) or train operating company (TOC). The general goal is a better timetable construction and the provision of a reliable train operation. This goal can be achieved with the RailSys simulation software. Over the last few years, the technology has been established that allows for the handling of very large networks and more than 10,000 trains in one single RailSys model [1,2]. Therefore, it is now possible to determine the effects of conflicts between trains, or the propagation of delays in networks of any size and complexity.

The operational simulation of railways supports this process. Predicting the robustness of a timetable is a critical issue for the future. With the help of 
RailSys both RC and TOC will be in the position to check a timetable before the timetable is operated in reality. A "simulation in reality" of a new timetable may contain a high risk for $\mathrm{RC}$ or $\mathrm{TOC}$ in the future due to penalties for poor OnTime-Running performance (OTR), or by jeopardising patronage or business from freight. In conclusion, the operational simulation of new timetables may become a "must" in the future and should be integrated into the workflow of the timetable production.

This article describes the fundamental aspects, the areas of application and some practical experiences. An outlook about some changes of processes and the latest developments in this context are given.

\section{Fundamentals}

The fundamental aspects of the state of the art timetable management and operational simulation are exemplified in the following paragraphs:

\subsection{Microscopic infrastructure model}

A node-link model describes the real infrastructure in a microscopic infrastructure model. All relevant information for planning a reliable timetable can be contained in such a model. The microscopic infrastructure model combines track information, such as speed, gradient, or radius, with the signalling system (type, blocks) and some operational information like routes or timing points.

Microscopic models are used for the operational planning of networks, lines and junctions. The modelling of infrastructure and operation in networks helps to avoid suboptimal solutions. The interrelation of the infrastructure, the rolling stock and the timetable should only be assessed on a network level. The complexity includes various signalling systems, different vehicle dynamics and train scheduling rules as well as additional operational conditions. For example, the identification and evaluation of reliable capacity indicators for railway lines require the inclusion of at least the adjacent junctions. It became apparent during the practical application of simulation methods that all branch lines of those junctions should be considered as well.

A simplified example in Figure 1 illustrates a transition between different infrastructure models. In a "Bottom Up" approach, it is evident that all macroscopic models can be derived in a straightforward manner from microscopic models. The "Top Down" approach can be used for generating microscopic models whose accuracy of detail depends on predefined assumptions. The "mesoscopic infrastructure model" has been placed in between both models. The models can be generated for specific tasks such as the simplified railway simulation of complex networks to answer some strategic questions. "Multi scale" models contain both areas that are modelled on a microscopic level and areas that are modelled on a macroscopic level. The advantage of such models is the minimisation of effort for modelling aspects of a complex problem that are of insignificant relevance for the over-all outcome of 
the investigation (e.g. the individual operation of shunting yards or vehicle depots for the planning of a network wide timetable).

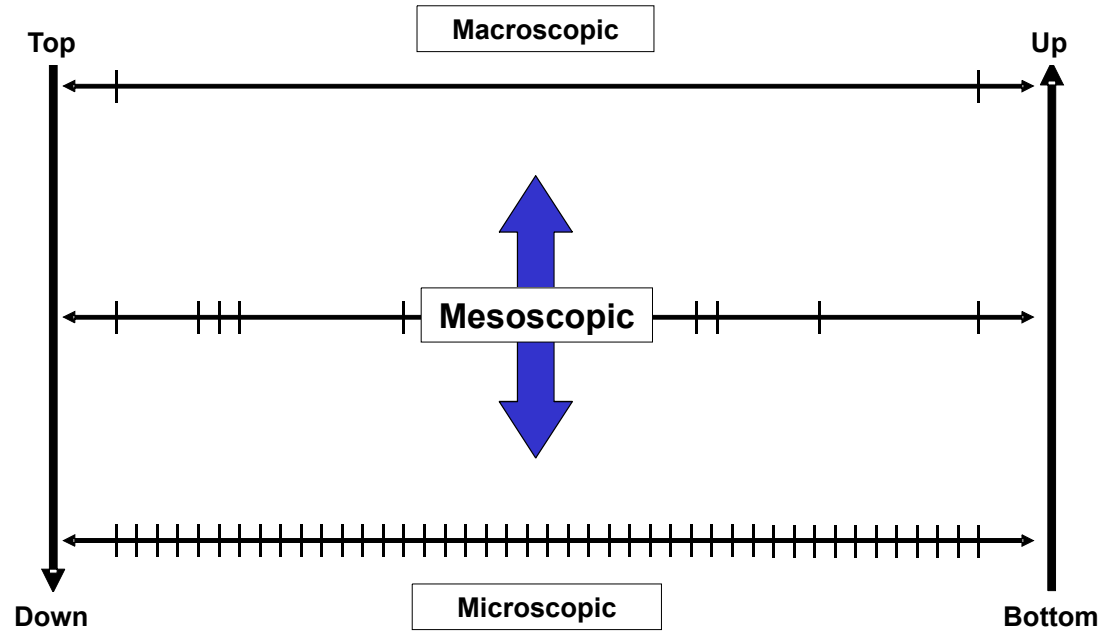

Figure 1: $\quad$ Multi scale infrastructure models.

It might be necessary to increase the level of detail of multi scaled infrastructure models for specific applications. This can be achieved without any problems, if the correct microscopic data is available. The further discussion in this article refers only to the microscopic models.

\subsection{Exact running time calculation}

The exact calculation of running times is essential for the correct planning of timetables. It should be possible to immediately recalculate running times at any time in microscopic models. For example, rerouting a train into another platform or changing the weight of a train may lead to significantly different running times. The variation of the running time may result in conflicts between trains, which need to be considered during the timetabling process. Using pre-processed sectional running times is, from our point of view, only a valid option if a microscopic infrastructure network for the timetable planning process is not available.

\subsection{Block occupation and conflict detection}

The microscopic description of the infrastructure model allows for the identification of conflicts and calculation of headways in the network, without regard to if only lines, junctions, or whole networks are considered. Figure 2 illustrates the principle: the horizontal axis is used for the geographical reference (signals, stations); the vertical axis represents the time. The "gradient" of the individual time-distance graphs reflects the speed of the trains. The block occupation boxes highlight the track occupation by a train. The resulting 
occupation depends on the signalling and block system. Additionally the minimum headways can be derived from the illustration, which provide a first indication of the track capacity.

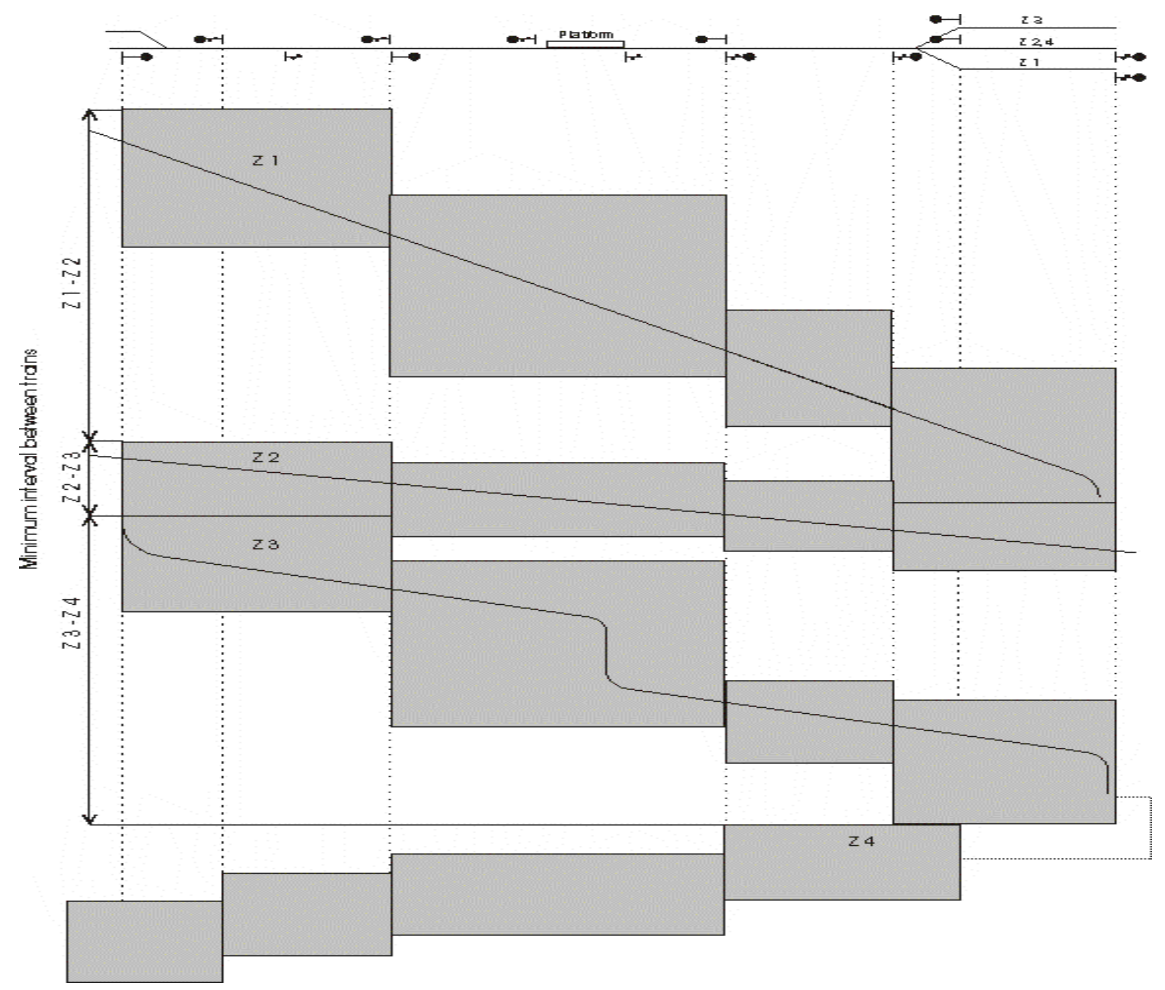

Figure 2: $\quad$ Block occupation [3].

\subsection{Operational synchronous simulation}

A synchronous simulation can be described as a dynamic process, in which all trains are running according to their schedule. Therefore, it is possible that some trains influence other trains, which results in delays or dispatching actions. In contrast, asynchronous methods handle all trains according to a hierarchy and assign trains one after the other into the model starting with high priority trains and ending with low priority trains. It is evident and has been proven that only the synchronous simulation models can provide indicators such as OTR performance reliably or accurately describes the delay propagation in railway networks. OTR values are very important for the design and operation of business rules and penalty regimes between RC and TOC.

The synchronous simulation can be used for two types of investigation. The first type is the simulation of a timetable to investigate the effects of inherent conflicts spreading throughout a network. During the timetable simulation there will be no introduction of additional delays, for example dwell time extensions. 
The second part is the operational simulation (formerly called stochastic or multiple simulation). The operational simulation includes the introduction of additional delays according to statistical rules. In general, between 50 and 200 timetable variations with different delays must be simulated to obtain a sufficient statistical basis for the evaluation. Both the timetable and operational simulation use powerful dispatching algorithms to reflect the real time dispatching [4].

The following list summarises the most important elements and advantages of the synchronous simulation, which result in a reliable representation of real world conditions and operation:

- All trains are simulated in parallel to realistically reflect the interaction between trains. Included in the simulation are deceleration and acceleration of trains due to conflicts between one another,

- Consideration of external influences such as passenger amount, driver response to signalling, or technical defects of signals, points, or rolling stock,

- Utilisation of dispatching algorithms for solving operational problems,

- Recording of all simulation data for subsequent evaluation.

\section{Determination of base characteristics for the evaluation of the performance}

Determination of base characteristics

1. Feasibility of a path

1.1 in one operating day

1.2 at a desired time

2. Transport

2.1 time

2.2 route

2.3 (speed)

3. Delay development

4. On-time

5. Connections

6. Vehicle rostering measured as/in

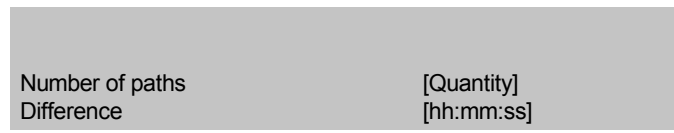

Transportation time [hh:mm:ss]

Transportation route [km]

Transportation speed [km/h]

Average delay [hh: $\mathrm{mm}: \mathrm{ss}]$

Delay frequency in classes [mm:ss]

Connections reached

[Number of days], [\%]

Train reversals (circulation)

[Number of days], [\%]

\section{ermcon}

Figure 3: $\quad$ Selection of key indicators for operational simulation.

\subsection{Evaluation of and key indicators for operational synchronous simulation}

The evaluation of the results of the operational simulation provides essential information about the advantages or disadvantages of a timetable or 
infrastructure concept. Some of the most important performance characteristics are displayed in Figure 3. The framework in which these key indicators fit may be different from $\mathrm{RC}$ to $\mathrm{RC}$. However, the main point is to prove the reliability of the railway operation.

It will be possible in the future to manage timetables in a more complex environment with the help of those key indicators. Operational requirements such as vehicle rostering or the possibility to keep connections for passengers will have an increased influence over the timetable construction in the future.

\section{Areas of application}

In general, we can distinguish between the following areas of application for the microscopic timetable simulation. Of course, there are tasks that cover more than one area.

\subsection{Long Term Planning (LTP)}

The LTP deals with the establishment of timetable concepts that are based on the demand prognosis for freight and passenger traffic for a time horizon of five and more years. The amalgamation of macroscopic and microscopic data models has recently been achieved by linking the Network Modelling macroscopic model (NEMO) with the RailSys microscopic model in Austria for the LTP. The operational concepts that are developed by NEMO are transformed into a timetable on a microscopic railway network. The transformation is performed with the RailSys slot search engine that is described in [4].

\subsection{Timetable design}

The core business of an $\mathrm{RC}$ is to provide the rail infrastructure and the planning competence to schedule trains. The scheduling of trains has to pay regards to the system immanent railborne character of railways traffic, which significantly restricts the flexibility of movements. Therefore, the timetable design is one of the most important tasks to be considered. The sequence of trains and the resulting timely order is predetermined by the timetable design and can hardly be altered at a later stage.

In general, all RC have to solve the problem of providing a maximum number of train paths (we may call it in a simplified way here "capacity") on a given infrastructure for a minimum of operational cost. In addition, political demands and requirements of TOC have to be taken into account, for example to operate passenger trains in rural areas even without gaining profit or covering the operational costs.

The timetable design itself is a highly dynamic process. It takes in general up to 24 months to design a timetable. The process involves various steps from the first draft to a working timetable. The requirements include the feeding of internal and public information systems or the provision of print outs for the operation. There are various iterations necessary to work out an "optimal" solution between all parties involved (RC, TOC and the market). In addition, the 
available budgets (often public money) will influence this process; competitors like the air sector have to be considered when planning a timetable.

\subsection{Short Term Planning (STP) and Ad-Hoc-Dispatching}

The simulation of railway operation can provide valuable assistance and support for both STP and ad-hoc dispatching.

STP may be required for operating a scheduled timetable with a shortage of suitable vehicles, or for planning urgent possessions for track work. It could be necessary to solve conflicts during the real time operation due to variations of train length, weight or locomotive supply. Temporary speed restrictions must also be catered for. Other specific situations may require urgent dispatching actions, for example bad weather or infrastructure failures. In all theses instances a methodology that is based on the above-described fundamentals is suitable for maintaining a reliable operation. Software such as RailSys, which clearly reflects those principles, is a most valuable tool.

\subsection{Vehicle allocation}

Like most of the afore mentioned tasks, the allocation of rolling stock is an essential part of the planning process. However, today there are in general two individual departments in railway organisations who deal separately with this issue. In general, it is a sequential process. The timetable design is followed by the vehicle allocation. RailSys offers an interactive interface to the Dispo software. The interface facilitates the export of the infrastructure network, the timetable, and other operational information that is essential for performing the vehicle allocation.

\subsection{Support for franchise bidding}

The preparation of franchise biddings in the UK and other countries with liberated railway networks is becoming an increasingly frequent task. Therefore, the process of timetable design and vehicle allocation needs to be performed quasi simultaneously. In the UK, it is possible to obtain a microscopic RailSys network model and existing timetable information from Network Rail under certain conditions. This fundamental information enables each franchise consortium to plan an exact timetable with correct running time calculation and conflict detection. Furthermore it is possible to predict the OTR of the planned timetable and therefore to reduce the risk of paying penalties for not achieving the required OTR performance.

\subsection{Possession planning}

The planning of possessions for the maintenance of existing railway networks will become a more important task. This is particularly true due to the decreasing budgets for new railway infrastructure in European countries with already 
established railway networks. It will be essential for the $\mathrm{RC}$ to provide reliable information to the TOC in time about alterations of the timetable due to changes in running times, which might be a consequence of track blockings or temporary speed restrictions.

For all these tasks, the synchronous simulation of microscopic infrastructure models can be seen as a powerful instrument for the control of the proposed plans. Furthermore, the slot search engine can be used during all planning stages. It provides additional support to the planner when creating new timetables or altering existing train runs.

\section{Practical experiences}

The Seven-Step-Model is derived from the experience that has been gained through the practical application of microscopic simulation models. This approach is the first step towards a comprehensive methodology on working with microscopic models. The principles are not limited to RailSys only; all synchronous simulation tools may be embedded in that methodology. In the following list the main steps are defined:

\section{Step Description}

I Definition of the project type

II Data acquisition and essential elements of the model

III Validation of the base model

IV Timetable design

V Timetable studies and slot management

VI Operational simulation

VII Evaluation and presentation of results

Figure 4 shows that it should be considered at the beginning of a project if software enhancements are useful or necessary. If software enhancements are required, special care should be taken for the specification, programming and testing of any software enhancements. However, software amendments are very rarely required.

The model calibration and validation takes place during steps III and VI. Those activities make the highest demands on user input and know how. At these points, the modelling techniques may have a certain influence on the workflow or even on the results. Modellers with experience cannot be replaced by software!

On the other hand, the modelling know how can be learned in a reasonable amount of time when the Seven-Step-Model is applied and projects are structured very well. A structured approach and knowledge transfer guarantee reliable results. The definition of rules for the modelling process or the application of a "best practise" can significantly support the transfer of knowledge. 


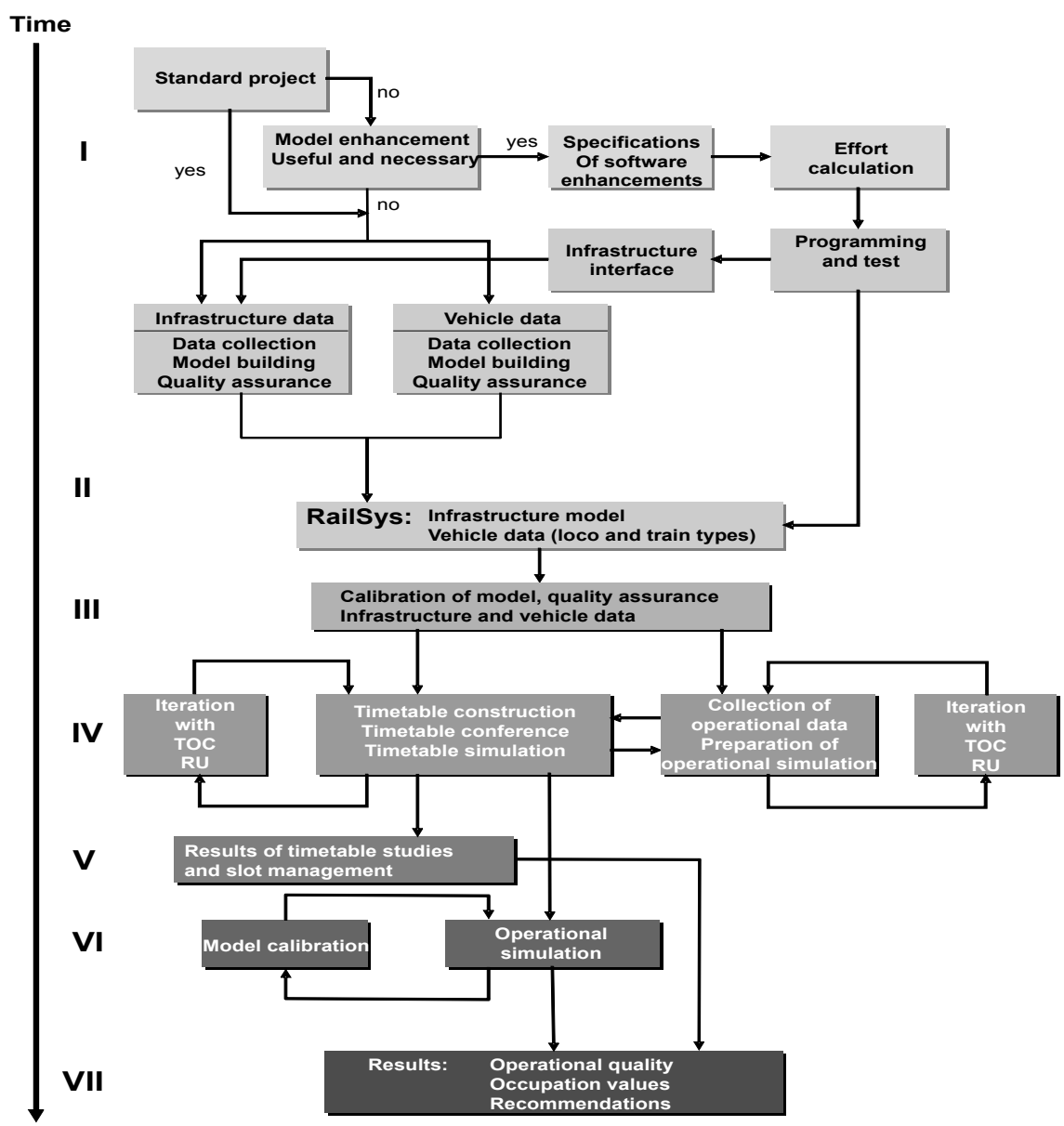

Figure 4: Seven-Step-Model [5].

\section{Perspectives of the planning process}

The future in the planning of railways may be illustrated in the following example. The illustration should be considered only as schematic. It does not illustrate or handle any details. This example considers only four activities or peaks during the planning process:

- Long Term Planning

- Timetable Construction

- Vehicle Allocation

- Possession planning

In general, for each of the four activities we can assume as a worst case that different teams are working with different tools on different databases. 
Furthermore, those teams may be assigned to different business units within one company or even in different companies. This situation may of course cause many redundancies in maintaining databases and organizing the workflow. The amount of resources for administration might be relatively high in order to manage this situation.

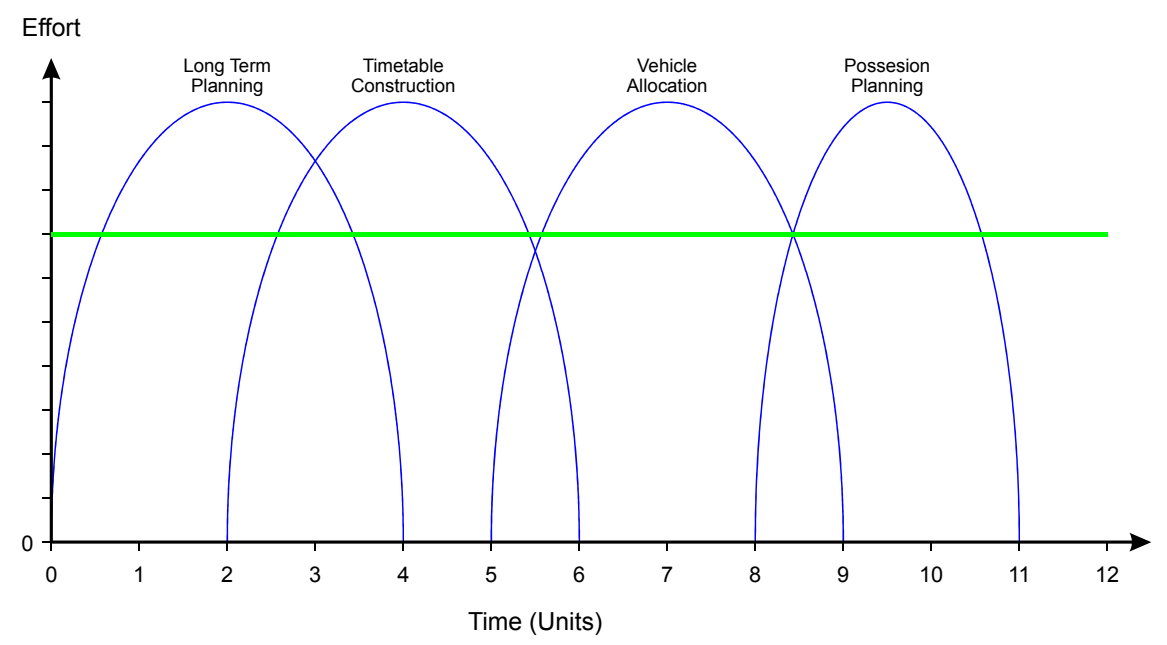

Figure 5: $\quad$ Future planning process.

In the future, very well educated expert teams will perform all four tasks in a more integrated and flexible way (indicated by the green line). From the technical point of view, a common microscopic database for all tasks will be the key issue. Microscopic data will increase the reliability of results and utilising the appropriate technology will result in rapid turn around times.

The use of clearly defined and permanently maintained interfaces for the data transfer will be essential. Probably only a few product oriented software tools will be in use in the future. These products will have to be very flexible. They will have a modular design that enables them to respond to different business demands. The advantage for RC and TOC then will be the possibility to reduce development costs significantly and to share the risks of any new development with several users.

As another result, any difference - if they ever exist - between "planning" and "production" tasks and systems will disappear because both tasks will amalgamate into each other. On the other hand, the complexity of all these processes will increase and will need excellent management.

\section{Summary}

The paper provides a short overview of the fundamental basics about which modelling techniques should be applied for the timetable design and the simulation of railway operation. The focus has been set on the microscopic 
infrastructure models pointing out new techniques of flexible infrastructure modelling with a "Multi Scale" approach.

The introduction of the Seven-Step-Model illustrates major steps of modelling. This model transferred some feedback from several hundred projects performed by IVE, RMCon and RailSys customers over recent years into the modelling process. The next challenges will be to find a straightforward way to establish microscopic modelling as the basis for all planning purposes. The impact of such an approach may be that very well educated expert teams will lead not only one process but several.

\section{References}

[1] http://www.rmcon.de

[2] http://www.ive.uni-hannover.de

[3] Siefer, Th., Radtke, A.: "Lectures Summer School Tongij University Shanghai, China 2005".

[4] Radtke, A., Hauptmann, D.: “Automated planning of timetables in large railway networks using a microscopic data basis and railway simulation techniques", Comprail Dresden 2004, Proceedings page 615 - 624

[5] Radtke, A.: "EDV-Verfahren zur Modellierung des Eisenbahnbetriebs", Habilitation an der Universität Hannover 2005 